\title{
Changes in adverse maternal, pregnancy, and delivery outcomes among U.S. delivery hospitalizations during the COVID-19 Pandemic, 2019-2020
}

\section{Regina M. Simeone ( $\square$ uzx8@cdc.gov )}

Centers for Disease Control and Prevention

\section{Karrie F. Downing}

Centers for Disease Control and Prevention

\section{Bailey Wallace}

Centers for Disease Control and Prevention

\section{Romeo R. Galang}

Centers for Disease Control and Prevention

\section{Carla L. DeSisto}

Centers for Disease Control and Prevention

\section{Van T. Tong}

Centers for Disease Control and Prevention

\section{Lauren B. Zapata}

Centers for Disease Control and Prevention and Public Health Service, Commissioned Corps Jean Y. Ko

Centers for Disease Control and Prevention and Public Health Service, Commissioned Corps

\section{Sascha R. Ellington}

Centers for Disease Control and Prevention

\section{Research Article}

Keywords: COVID-19, Pregnancy outcome, Delivery outcome, Preterm birth, Obstetric delivery

Posted Date: September 30th, 2021

DOI: https://doi.org/10.21203/rs.3.rs-949566/v1

License: (c) (1) This work is licensed under a Creative Commons Attribution 4.0 International License. Read Full License 


\section{Abstract \\ Background}

Early reports suggested changes in select pregnancy outcomes during the coronavirus disease 2019 (COVID-19) pandemic. The population-level impact of the pandemic on pregnancy and delivery outcomes is not well understood.

\section{Objective}

To assess whether pregnancies that ended during the pandemic were at increased or decreased risk of selected adverse outcomes compared to the previous year.

\section{Methods}

Data from a cohort of delivery hospitalizations occurring during April-December of 2019 and 2020 in the Premier Healthcare Database were analyzed. Prevalence of select adverse outcomes were examined. Adjusted prevalence ratios (aPR) and 95\% confidence intervals (Cl) were estimated using Poisson regression with robust standard errors.

\section{Results}

We identified 663,620 deliveries occurring April-December of 2019 and 614,093 deliveries occurring AprilDecember of 2020. We observed an increase in in-hospital death from 2019 to 2020 ( $n=33 ; 5$ per 100,000 vs. $n=53 ; 9$ per 100,000 , aPR: $1.68, \mathrm{Cl}: 1.09,2.59)$, which was no longer statistically significant after excluding deliveries with a COVID-19 diagnosis (aPR: $1.25, \mathrm{Cl}: 0.78,2.00)$. Intensive care unit admission decreased from 2019 to 2020 ( $n=10,964 ; 1.7 \%$ vs. $n=9,480 ; 1.5 \%$, aPR: 0.90, Cl: 0.87, 0.92). Preterm birth decreased from 2019 to 2020 ( $n=61,101 ; 9.2 \%$ vs. $n=56,158 ; 9.1 \%$, aPR: 0.96, Cl: 0.95, 0.97). The prevalence of cesarean deliveries was similar in 2019 and 2020 (30.9\% vs 31.6\%, respectively), but there were some differences in cesarean delivery characteristics (e.g., an increase in cesarean deliveries due to conduct of labor $[n=2,553 ; 1.2 \%$ vs. $n=2,642 ; 1.4 \%$, aPR: 1.11, Cl: 1.05, 1.17]) from 2019 to 2020.

\section{Conclusions}

In 2020 compared to 2019, in-hospital deaths among pregnant women increased and there was a small decrease in prevalence of preterm births. The full impact of the COVID-19 pandemic on maternal and pregnancy outcomes remains to be understood. However, it appears that most outcomes investigated experienced minimal change from 2019 to 2020. 


\section{Background}

The immense scale of the coronavirus disease 2019 (COVID-19) pandemic has resulted in substantial changes to healthcare delivery throughout the United States, including reductions in the use of preventive care and other health services. ${ }^{1,2}$ The delivery of obstetric care, including prenatal and intrapartum services, has also been impacted. ${ }^{3-5}$ Stay-at-home orders that were declared across the country in 2020 and intended to mitigate spread of the virus required abrupt changes to individuals' work, social, and physical activity habits. Additionally, social isolation and increased caregiving responsibilities have been identified as potential negative influences impacting health. ${ }^{6-9}$ Changes in healthcare policies, utilization, and access, as well as increased time at home, stress, and mental health concerns could impact the health and healthcare of pregnant people.

Compared to pregnant persons without severe acute respiratory syndrome coronavirus 2 (SARS-CoV-2) infection, the virus that causes COVID-19, pregnant persons with SARS-CoV-2 are at increased risk of preterm birth and may be at increased risk of other adverse pregnancy outcomes, including stillbirth. ${ }^{10-14}$ Further, pregnant and recently pregnant persons with SARS-CoV-2 infection are at increased risk of severe COVID-19 illness, compared to non-pregnant people. ${ }^{12,15}$ Less is known about whether non-COVID-19 maternal outcomes are different than before the pandemic. Early in the pandemic, reports from outside of the United States detailed reductions in preterm birth and low birthweight infants after the implementation of COVID-19 mitigation measures, including stay-at-home orders. ${ }^{16-20}$ However, results of U.S. studies assessing the impact of COVID-19 mitigation measures on preterm birth have been mixed. ${ }^{21-25}$ Pandemic-related changes in adverse maternal and pregnancy outcomes may vary based on country-level resources and within-state differences. For example, a recent systematic review observed reductions in preterm birth only in high-income countries, whereas significant increases in maternal and fetal mortality were more often observed in low-income and middle-income countries. ${ }^{21-26}$

Within the context of the COVID-19 pandemic, many hospitals implemented infection control measures to help protect hospital staff, patients, and caregivers from contracting COVID-19.4, 27, 28 Measures included SARS-CoV-2 screening for women upon hospital admission, restricting the number of support persons and visitors for laboring women, and policies for masks and personal protective equipment in healthcare facilities. Additionally, the American College of Obstetricians and Gynecologists (ACOG) provided guidance clarifying that SARS-CoV-2 infection is not an indication for cesarean delivery and that labor induction and cesarean should be based on obstetric indications and not COVID-19 status. ${ }^{4}$ ACOG additionally recommended expedited discharge of mother-infant dyads when appropriate to help prevent SARS-CoV-2 infection during the delivery hospitalization. ${ }^{4}$

The collective impact of the COVID-19 pandemic, including mitigation measures, on being pregnant and on the delivery of obstetric care is not well understood. Previous U.S. studies have been limited in the outcomes they could observe or only assessed outcomes in single hospital centers. Thus, using data 
from a large multi-state U.S. hospital-based administrative database, we compared the prevalence of selected maternal, delivery, and pregnancy outcomes occurring in 2020 to those in 2019.

\section{Methods \\ 2.1 Data}

We performed a cohort analysis of delivery hospitalizations using the Premier Healthcare Database Special COVID-19 Release (https://www.premierinc.com/; PHD-SR, release date 8/15/2021). The PHD-SR is a U.S. hospital-based, service-level, all-payor database. It represents approximately $20 \%$ of all U.S. inpatient admissions, regardless of payor type, from geographically diverse non-profit, non-governmental, community, and teaching hospitals and health systems from rural and urban areas. ${ }^{29}$ We included hospitals that contributed data for at least one maternal delivery discharge during April-December in both 2019 and 2020 and restricted the sample to the same hospitals in each year.

\subsection{Covariates}

Delivery hospitalizations were identified among women aged 15-45 years using International Classification of Diseases, 10th revision, Clinical Modification (ICD-10-CM) diagnostic and procedure codes, as well as diagnostic related group delivery codes, for singleton livebirth or stillbirth deliveries on the patient diagnosis and procedure tables (Appendix 1). Deliveries with codes for multiple births were excluded due to the association between multiple gestation and preterm delivery. Deliveries were limited to those discharged from April 1st - December 31st in 2020 or in 2019. Gestational age at delivery was assessed using Z3A.xx codes; pregnancies that ended prior to 20 weeks gestation or after 42 weeks gestation were excluded. If a woman had more than one delivery hospitalization coded as occurring in the same year, with hospitalizations greater than 30 days apart or with a difference in estimated gestational age that changed the classification of preterm vs. term birth, she was excluded from the analysis ( $n=239$ women/485 encounters in 2019 and 183 women/375 encounters in 2020). If a woman had more than one delivery hospitalization coded the same year, but with hospitalizations less than or equal to 30 days apart and with the same preterm/term categorization, the first delivery hospitalization encounter was included, and subsequent encounters were excluded ( $n=1,114$ women contributing 1,127 excluded encounters in 2019 and 1,060 women contributing 1,070 encounters in 2020).

Maternal demographic characteristics included maternal age, race and ethnicity, marital status, and primary payor. Maternal race and ethnicity are collected separately in the PHD-SR and were combined for this analysis. Women with records indicating Hispanic ethnicity were considered Hispanic, regardless of recorded or missing race. Women missing Hispanic ethnicity or categorized as non-Hispanic were classified as non-Hispanic and assigned their recorded race. Race was missing in $3 \%$ of observations. We additionally considered U.S. Census division location and urban/rural status as hospital characteristics.

We identified the following maternal comorbidities, which are associated with adverse pregnancy outcomes and increased risk of cesarean delivery: hypertensive disorders (chronic hypertension, 
gestational hypertension, chronic hypertension with superimposed pre-eclampsia, pre-eclampsia, Hemolysis, elevated liver enzymes, low platelet count (HELLP) syndrome, and eclampsia), diabetes (Type 1, Type 2, gestational, other/unspecified), and obesity (Appendix 2).

\subsection{Outcomes}

We identified selected adverse maternal and pregnancy outcomes if an ICD-10-CM code was present on the delivery hospitalization record. Our primary outcomes were maternal intensive care unit (ICU) admission, in-hospital death, preterm birth, and stillbirth. Maternal ICU admission was identified in the hospital chargemaster records, and in-hospital death was identified using the maternal discharge status code (Appendix 3). Pregnancies with a gestational age of less than 37 weeks were considered preterm; pregnancies with a gestational age of 37 to 42 weeks were considered term.

Secondary outcomes included mode of delivery (vaginal or cesarean), and cesarean delivery characteristics. Among cesarean deliveries, we identified those with premature rupture of membranes (PROM), prolonged labor, attempted assisted vaginal delivery by forceps or vacuum, or augmentation or induction of labor (Appendix 3). These classifications were not mutually exclusive. We further identified cesarean deliveries considered to be at low-risk for cesarean delivery using the Society for Maternal-Fetal Medicine (SMFM) updated definition. ${ }^{30}$ Those excluded from the low-risk category (hereafter referred to as high-risk) were further categorized into the following non-mutually exclusive groups: maternal factors (e.g., HIV disease, eclampsia, congenital cardiovascular disorders of mother), preterm birth, stillborn, malpresentation (e.g., breech presentation), fetal factors (e.g., hydrocephalic fetus), uterine/placental factors (e.g., placenta previa, previous cesarean delivery), and conduct of labor (e.g., failed forceps or vacuum extraction) (Appendix 3).

Finally, we examined maternal inpatient readmission to the same hospital within 30 days. We additionally examined the overall distribution of delivery length of stay (vaginal: $<1$ day, 1 day, 2 days, $\geq 3$ days; cesarean: $<1$ day, $1-2$ days, 3 days, 4 days, $\geq 5$ days), stratified by mode of delivery.

\subsection{Analysis}

We report the distribution of maternal and hospital characteristics and adverse maternal and pregnancy outcomes in 2020 compared to 2019. Poisson regression models, with robust standard errors, were used to estimate unadjusted and adjusted prevalence ratios (aPRs) for adverse maternal and pregnancy outcomes comparing those occurring in 2020 to those occurring in 2019. ${ }^{31}$ For the assessment of delivery length of stay, we assessed the overall differences in the distribution of length of stay in 2019 and 2020 using a chi square test. We also ran separate Poisson regression models, comparing each category of length of stay (defined above) to a reference category (vaginal deliveries: two days; cesarean deliveries: three days). Data recorded in unique hospitals may be correlated; additionally, 20,458 women delivered in both 2019 and 2020. We accounted for correlations within individuals, nested within hospitals, using an exchangeable correlation matrix. We considered the following covariates: maternal age, census division, primary payor, and maternal comorbidities. Models were adjusted for different 
subsets of covariates, depending on the outcome and ability to achieve model convergence. As a sensitivity analysis, we excluded women with a COVID-19 diagnosis (ICD-10-CM code U07.1 from April 2020-December 2020 or B97.29 from March-April 2020) ${ }^{32}$ at the delivery hospitalization to assess if the direct effect of SARS-CoV-2 infection during pregnancy was the primary contributor to differences in adverse outcomes. As a second sensitivity analysis, we stratified our analyses by quarter (Quarter 2: AprilJune; Quarter 3: July-September; Quarter 4: October-December) to assess whether changes in selected outcomes differed over the course of the pandemic compared to the same time period the previous year. Because analyses of large datasets can result in statistically significant findings that may not be meaningful, we a priori chose to report results of cesarean delivery characteristics if they had an aPR indicating at least $5 \%$ change in 2020 compared to 2019 . This activity was reviewed by CDC and was conducted consistent with applicable federal law and CDC policy; the activity was determined to meet the requirements of public health surveillance as defined in 45 CFR 46.102(I)(2).

\section{Results}

There were 664 hospitals that reported at least one delivery discharged during April through December in both 2019 and 2020. We identified 663,620 deliveries occurring during April through December of 2019 and 614,093 deliveries occurring during April through December of 2020 from these hospitals (Table 1, Figure 1). Maternal demographic characteristics were similar in 2019 and 2020, with slightly more deliveries in 2020 occurring among women of Hispanic ethnicity, and slightly fewer deliveries in 2020 occurring among married women. Hospital characteristics were also similar in 2019 and 2020. The presence of maternal medical conditions documented at delivery increased across conditions studied from 2019 to 2020. The increase in diabetes appeared to be driven by an increase in gestational diabetes from $8.4 \%$ in 2019 to $9.8 \%$ in 2020 .

Slightly fewer deliveries resulted in ICU admission in 2020 compared to 2019 (1.5\% vs 1.7\%, respectively, aPR $=0.90, \mathrm{Cl}: 0.88,0.92)$. Deliveries in 2020 were more likely to result in in-hospital death (aPR=1.68, Cl: $1.09,2.59)$, though absolute risk of death was based on few events (2019: $n=33,5$ per 100,000; 2020 : $n=53,9$ per 100,000) (Table 2). When women with COVID-19 at delivery were removed from the analysis,

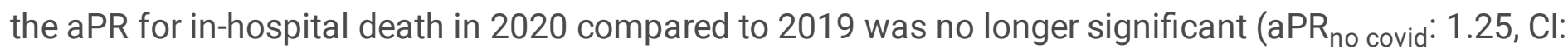
$0.78,2.00$ ) (Appendix 4). We did not observe differences in the prevalence of livebirths or stillbirths from 2019 to 2020 . The prevalence of preterm birth decreased slightly from 2019 to 2020 (9.2\% vs. 9.1\%, aPR $=0.96, \mathrm{Cl}: 0.95,0.97)$. There was no change in the prevalence of cesarean delivery from 2019 to 2020 (30.9\% vs. $31.6 \%$, aPR $=1.00, \mathrm{Cl}: 1.00,1.01)$. We observed increases of $\geq 5 \%$ for cesarean deliveries with PROM (aPR: 1.06, Cl: 1.03, 1.08), prolonged labor (aPR: $1.06(1.00,1.15)$, and attempted forceps or vacuum (aPR: $1.06, \mathrm{Cl}: 0.98,1.15)$. We additionally observed increases in cesarean deliveries considered high-risk due to maternal factors (aPR: 1.07, Cl: 1.03, 1.12) and conduct of labor (aPR: 1.11, Cl: 1.05, 1.17).

The overall distribution of delivery length of stay differed in 2020 compared to 2019 (Figure 2, $p<0.0001$ ), with a greater proportion of shorter hospital stays occurring after vaginal and cesarean deliveries in 2020 
compared to 2019. Further, among vaginal deliveries, after adjusting for within individual and hospital correlations, as well as maternal and hospital characteristics, delivery length of stays less than two days were more common in 2020 compared to 2019 (Table 3). Similarly, among cesarean deliveries, delivery length of stays less than three days were more common in 2020 compared to 2019.

Most results did not substantially differ after excluding women with a COVID-19 diagnosis at delivery ( $n=11,0951.8 \%$ of 2020 deliveries). When we stratified the analysis by quarter, the distribution of maternal and hospital characteristics was similar to those observed in the main analysis (Appendix 5). Overall, the adverse maternal and pregnancy outcomes were similar by quarter, with overlapping $95 \% \mathrm{Cls}$ (Appendices 6-7). The aPR for ICU admission and preterm birth was lowest in the second and third quarters, but became null by the fourth quarter. Similarly, in the second quarter, the adjusted prevalence of readmission within 30 days of delivery was $15 \%$ lower in 2020 compared to $2019\left(\mathrm{aPR}_{\mathrm{Q} 2}=0.86,95 \% \mathrm{Cl}\right.$ : 0.81-0.91); however, the same association was approximately null and no longer significant in the latter two quarters of 2020 (Appendix 7). Quarterly associations between delivery length of stay in 2020 compared to 2019 were similar to the main analysis (Appendix 8).

\section{Comment}

\subsection{Principal findings}

In this analysis comparing deliveries occurring during April through December of 2019 and 2020, we observed statistical differences in delivery outcomes or mode of delivery and complications, but it is unclear whether many of these differences are clinically relevant. The prevalence of preterm birth decreased slightly in the last three quarters of 2020 compared to 2019 . The overall change in prevalence was less than $5 \%$, but the decrease is consistent with national estimates. ${ }^{33}$ Similarly, cesarean deliveries with PROM, prolonged labor, and attempted forceps or vacuum increased in 2020 compared to 2019, with small absolute and relative differences. Cesarean deliveries considered high-risk due to maternal conditions and conduct of labor increased in 2020 compared to 2019. Among all pregnancies, delivery length of stay was shorter for deliveries in 2020 compared to 2019, likely due to COVID-19 mitigation measures. Among all women included in our sample, in-hospital death increased 69\% from 2019 to 2020, although absolute risk was less than $0.01 \%$ in both years. However, in the sensitivity analysis that excluded women with a COVID-19 diagnosis at delivery from the 2020 sample, the association was no longer significant. These results support the hypothesis that COVID-19 likely drove the increase in inhospital deaths, but further exploration of this outcome, with in-depth reviews from maternal mortality review committees, is needed to elucidate the cause of deaths. The sensitivity analysis assessing differences by quarter suggests that overall, outcomes were stable throughout 2020; the exceptions were preterm birth, which decreased early in the pandemic but became null in the latter half of the year, and readmission with 30 days of the delivery hospitalization, which followed a similar pattern.

\subsection{Limitations of the data}


The findings of this analysis should be interpreted considering several limitations. First, we used ICD-10$\mathrm{CM}$ codes in administrative discharge data to identify delivery, pregnancy, and maternal outcomes, which may have resulted in misclassification of outcomes, including COVID-19 diagnosis. ICD-10-CM codes do not provide information on COVID-19 symptom severity. Second, among women with multiple delivery hospitalizations separated by 30 days or less, the first delivery hospitalization encounter was used to determine adverse maternal and pregnancy outcomes; this could have resulted in misclassification of outcomes if the subsequent hospitalizations differed in recorded diagnoses. Third, because our analysis was limited to delivery hospitalizations and linkage across hospitals is not available, we did not have information on maternal health during pregnancy, including COVID-19 diagnosis during pregnancy, maternal pregnancy history (including prior cesarean delivery), maternal use of prenatal care, or hospitalizations occurring outside of the delivery hospital after pregnancy. We were also unable to link maternal and infant outcomes; thus, this analysis is focused only on selected maternal, delivery, and pregnancy outcomes present at the delivery hospitalization. Fourth, although we have a large population of delivery hospitalizations across the United States, the Premier data are not nationally representative. Fifth, although the Premier data include information on potential confounders, unmeasured confounding may exist. Finally, this analysis only included one year of baseline data to compare to outcomes in 2020, which could impact our results. It is possible that analyses incorporating additional years of data may yield different results.

\subsection{Strengths of the study}

Despite these limitations, our analysis had several strengths. The PHD-SR data are geographically diverse, allowing us to include hospitalizations from across the United States. Additionally, our population is racially diverse and represents deliveries occurring among multiple payor types. The large sample size allowed investigation of multiple pregnancy outcomes, mode of delivery and complications, and maternal outcomes that have not been well studied during the COVID-19 pandemic.

\subsection{Interpretation}

Our estimates of changes in pregnancy outcomes and mode of delivery adds to the literature of the impact of the COVID-19 pandemic on pregnancy outcomes. While some U.S. and European studies have observed reductions in preterm birth, ${ }^{16-18,20,21,24}$ others have not. ${ }^{22,23,25,34}$ Our data suggest that, after adjustment, pregnancies delivering in the last three quarters of 2020 were slightly less likely to deliver preterm compared to those delivering in 2019. The reduction in the absolute prevalence of preterm birth from 2019 to 2020 was small. However, this change in prevalence represents approximately 382 fewer preterm births occurring in 2020 than would be expected using the prevalence from 2019. Infants born preterm can experience long-term health problems, and costs of caring for children born preterm in the United States are substantial. ${ }^{35}$ Even modest reductions in preterm birth may have important public health implications. Some studies have observed increases in stillbirth rates during the pandemic. ${ }^{13,14}$

However, other evidence suggests that stillbirth may not be increased during the pandemic period. ${ }^{20,23,36}$ Our data do not suggest an increase in stillbirths during the COVID-19 pandemic. 
Our assessment of mode of delivery and characteristics of cesarean deliveries provides evidence that the COVID-19 pandemic has not substantially changed cesarean delivery practices in the hospitals included in this analysis, in accordance with ACOG recommendations. ${ }^{4}$ Additionally, the lack of difference between the prevalence of cesarean deliveries in 2020 compared to 2019 is consistent with what has been observed within the U.S. and other countries. ${ }^{20,33,36-38}$ Further, the shortened delivery hospitalization lengths of stay are also consistent with the literature and ACOG recommendations. ${ }^{4,39}$ The impact of shortened delivery hospitalizations on postpartum health remains to be studied; in our analytic sample, the prevalence of maternal hospital readmission within 30 days slightly decreased from 2019 to 2020 .

\section{Conclusions}

During the COVID-19 pandemic, fewer deliveries were preterm compared to women delivering during the same period in 2019. However, this reduction was only observed early in the pandemic. No changes in stillbirth were observed. The proportion of cesarean deliveries with PROM, prolonged labor, and attempted forceps or vacuum increased, as did cesarean deliveries with selected high-risk characteristics. We observed an increase in in-hospital death in 2020 compared to 2019, which should be further investigated. Of note, excluding women with COVID-19 diagnosis at delivery resulted in a difference that was not statistically significant. Future research should focus on identifying factors (e.g., COVID-19 mitigation measures, changes in clinical practices, stress, etc.) that contributed to these changes in pregnancy outcomes.

\section{Declarations}

\section{Acknowledgements}

John House, Premier Inc. for collecting, managing, and granting access to the dataset; CDC COVID-19 Response Data, Analytics and Visualization Task Force for data acquisition and analytic guidance; CDC COVID-19 Response Epidemiology Task Force for scientific guidance.

Conflicts of interest: The authors do not have any potential conflicts of interest to declare.

\section{References}

1. Whaley CM, Pera MF, Cantor J, Chang J, Velasco J, Hagg HK, et al. Changes in health services use among commercially insured US populations during the COVID-19 pandemic. JAMA Netw Open. 2020; 3:e2024984.

2. Hartnett KP, Kite-Powell A, DeVies J, Coletta MA, Boehmer TK, Adjemian J, et al. Impact of the COVID19 pandemic on emergency department visits - United States, January 1, 2019-May 30, 2020. MMWR Morb Mortal Wkly Rep. 2020; 69:699-704. 
3. Fryer K, Delgado A, Foti T, Reid CN, Marshall J. Implementation of obstetric telehealth during COVID19 and beyond. Matern Child Health J. 2020; 24:1104-1110.

4. The American College of Obstetricians and Gynecologists. COVID-19 FAQs for obstetriciansgynecologists, obstetrics. Washington, DC2020 [June 4, 2021]; Available from: https://www.acog.org/clinical-information/physician-faqs/covid-19-faqs-for-ob-gyns-obstetrics.

5. Centers for Disease Control and Prevention. Considerations for inpatient obstetric healthcare settings. 2020 [June 4, 2021]; Available from: https://www.cdc.gov/coronavirus/2019ncov/hcp/inpatient-obstetric-healthcare-guidance.html.

6. Tull MT, Edmonds KA, Scamaldo KM, Richmond JR, Rose JP, Gratz KL. Psychological outcomes associated with stay-at-home orders and the perceived impact of COVID-19 on daily life. Psychiatry Res. 2020; 289:113098.

7. Flanagan EW, Beyl RA, Fearnbach SN, Altazan AD, Martin CK, Redman LM. The impact of COVID-19 stay-at-home orders on health behaviors in adults. Obesity (Silver Spring). 2021; 29:438-445.

8. Moreland A, Herlihy C, Tynan MA, Sunshine G, McCord RF, Hilton C, et al. Timing of state and territorial COVID-19 stay-at-home orders and changes in population movement - United States, March 1-May 31, 2020. MMWR Morb Mortal Wkly Rep. 2020; 69:1198-1203.

9. Marroquin B, Vine V, Morgan R. Mental health during the COVID-19 pandemic: Effects of stay-athome policies, social distancing behavior, and social resources. Psychiatry Res. 2020; 293:113419.

10. Delahoy MJ, Whitaker M, O'Halloran A, Chai SJ, Kirley PD, Alden N, et al. Characteristics and maternal and birth outcomes of hospitalized pregnant women with laboratory-confirmed COVID-19 - COVIDNET, 13 States, March 1-August 22, 2020. MMWR Morb Mortal Wkly Rep. 2020; 69:1347-1354.

11. Ko JY, DeSisto CL, Simeone RM, Ellington S, Galang RR, Oduyebo T, et al. Adverse pregnancy outcomes, maternal complications, and severe illness among U.S. delivery hospitalizations with and without a COVID-19 diagnosis. Clin Infect Dis. 2021.

12. Zambrano LD, Ellington S, Strid P, Galang RR, Oduyebo T, Tong VT, et al. Update: Characteristics of symptomatic women of reproductive age with laboratory-confirmed SARS-CoV-2 infection by pregnancy status - United States, January 22-October 3, 2020. MMWR Morb Mortal Wkly Rep. 2020; 69:1641-1647.

13. Khalil A, von Dadelszen P, Draycott T, Ugwumadu A, O'Brien P, Magee L. Change in the incidence of stillbirth and preterm delivery during the COVID-19 pandemic. JAMA. 2020.

14. De Curtis M, Villani L, Polo A. Increase of stillbirth and decrease of late preterm infants during the COVID-19 pandemic lockdown. Arch Dis Child Fetal Neonatal Ed. 2020.

15. Allotey J, Stallings E, Bonet M, Yap M, Chatterjee S, Kew T, et al. Clinical manifestations, risk factors, and maternal and perinatal outcomes of coronavirus disease 2019 in pregnancy: living systematic review and meta-analysis. BMJ. 2020; 370:m3320.

16. Hedermann G, Hedley PL, Baekvad-Hansen M, Hjalgrim H, Rostgaard K, Poorisrisak P, et al. Danish premature birth rates during the COVID-19 lockdown. Arch Dis Child Fetal Neonatal Ed. 2021; 106:93-95. 
17. Been JV, Burgos Ochoa L, Bertens LCM, Schoenmakers S, Steegers EAP, Reiss IKM. Impact of COVID19 mitigation measures on the incidence of preterm birth: a national quasi-experimental study. Lancet Public Health. 2020; 5:e604-e611.

18. Matheson A, McGannon CJ, Malhotra A, Palmer KR, Stewart AE, Wallace EM, et al. Prematurity Rates During the Coronavirus Disease 2019 (COVID-19) Pandemic Lockdown in Melbourne, Australia. Obstet Gynecol. 2021; 137:405-407.

19. Philip RK, Purtill H, Reidy E, Daly M, Imcha M, McGrath D, et al. Unprecedented reduction in births of very low birthweight (VLBW) and extremely low birthweight (ELBW) infants during the COVID-19 lockdown in Ireland: a 'natural experiment' allowing analysis of data from the prior two decades. BMJ Glob Health. 2020; 5.

20. McDonnell S, McNamee E, Lindow SW, O'Connell MP. The impact of the Covid-19 pandemic on maternity services: A review of maternal and neonatal outcomes before, during and after the pandemic. Eur J Obstet Gynecol Reprod Biol. 2020; 255:172-176.

21. Harvey EM, McNeer E, McDonald MF, Shapiro-Mendoza CK, Dupont WD, Barfield W, et al. Association of preterm birth rate with COVID-19 statewide stay-at-home orders in Tennessee. JAMA Pediatr. 2021.

22. Main EK, Chang SC, Carpenter AM, Wise PH, Stevenson DK, Shaw GM, et al. Singleton preterm birth rates for racial and ethnic groups during the coronavirus disease 2019 pandemic in California. Am J Obstet Gynecol. 2021; 224:239-241.

23. Handley SC, Mullin AM, Elovitz MA, Gerson KD, Montoya-Williams D, Lorch SA, et al. Changes in preterm birth phenotypes and stillbirth at 2 Philadelphia hospitals during the SARS-CoV-2 pandemic, March-June 2020. JAMA. 2021; 325:87-89.

24. Berghella V, Boelig R, Roman A, Burd J, Anderson K. Decreased incidence of preterm birth during coronavirus disease 2019 pandemic. Am J Obstet Gynecol MFM. 2020; 2:100258.

25. Wood R, Sinnott C, Goldfarb I, Clapp M, McElrath T, Little S. Preterm birth during the coronavirus disease 2019 (COVID-19) pandemic in a large hospital system in the United States. Obstet Gynecol. 2021; 137:403-404.

26. Chmielewska B, Barratt I, Townsend R, Kalafat E, van der Meulen J, Gurol-Urganci I, et al. Effects of the COVID-19 pandemic on maternal and perinatal outcomes: a systematic review and metaanalysis. Lancet Glob Health. 2021; 9:e759-e772.

27. Boelig RC, Manuck T, Oliver EA, Di Mascio D, Saccone G, Bellussi F, et al. Labor and delivery guidance for COVID-19. Am J Obstet Gynecol MFM. 2020; 2:100110.

28. Society for Maternal-Fetal Medicine and Society for Obstetric and Anesthesia and Perinatology. Labor and delivery COVID-19 considerations. 2020 [June 4, 2021]; Available from: https://s3.amazonaws.com/cdn.smfm.org/media/2277/SMFM-SOAP_COVID_LD_Considerations_327-20_(final)_PDF.pdf.

29. Premier Healthcare Database white paper: Data that informs and performs. Premier; [updated Mar. 2, 2020; June 4, 2021]; Available from: http://offers.premierinc.com/rs/381-NBB- 
525/images/PHD_COVID-19_White_Paper.pdf.

30. Armstrong J, McDermott P, Saade GR, Srinivas SK, Society for Maternal-Fetal Medicine Health P, Advocacy $\mathrm{C}$, et al. Coding update of the SMFM definition of low risk for cesarean delivery from ICD-9CM to ICD-10-CM. Am J Obstet Gynecol. 2017; 217:B2-B12 e56.

31. Zou G. A modified poisson regression approach to prospective studies with binary data. Am J Epidemiol. 2004; 159:702-706.

32. Centers for Disease Control and Prevention. New ICD-10-CM code for the 2019 novel coronavirus (COVID-19), April 1, 2020. 2020 [June 4, 2021]; Available from:

https://www.cdc.gov/nchs/data/icd/Announcement-New-ICD-code-for-coronavirus-3-18-2020.pdf..

33. Hamilton B, Martin J, Osterman M. Births: Provisional data for 2020. Vital Statistics Rapid Release: National Center for Health Statistics. May 2021.

34. Son M, Gallagher K, Lo JY, Lindgren E, Burris HH, Dysart K, et al. Coronavirus disease 2019 (COVID19) pandemic and pregnancy outcomes in a U.S. population. Obstet Gynecol. 2021.

35. Frey HA, Klebanoff MA. The epidemiology, etiology, and costs of preterm birth. Semin Fetal Neonatal Med. 2016; 21:68-73.

36. Meyer R, Bart Y, Tsur A, Yinon Y, Friedrich L, Maixner N, et al. A marked decrease in preterm deliveries during the coronavirus disease 2019 pandemic. Am J Obstet Gynecol. 2021; 224:234-237.

37. Greene NH, Kilpatrick SJ, Wong MS, Ozimek JA, Naqvi M. Impact of labor and delivery unit policy modifications on maternal and neonatal outcomes during the coronavirus disease 2019 pandemic. Am J Obstet Gynecol MFM. 2020; 2:100234.

38. Bhatia K, Columb M, Bewlay A, Eccles J, Hulgur M, Jayan N, et al. The effect of COVID-19 on general anaesthesia rates for caesarean section. A cross-sectional analysis of six hospitals in the north-west of England. Anaesthesia. 2021; 76:312-319.

39. Bornstein E, Gulersen M, Husk G, Grunebaum A, Blitz MJ, Rafael TJ, et al. Early postpartum discharge during the COVID-19 pandemic. J Perinat Med. 2020; 48:1008-1012.

\section{Tables}

Table 1. Maternal and hospital characteristics of deliveries occurring from April through December of 2019 and 2020, Premier Healthcare Database Special COVID-19 Release, United States 


\begin{tabular}{lrrrr}
\hline & 2019 & 2020 & \\
\hline Characteristics & $\mathrm{n}$ & $\%$ & $\mathrm{n}$ & $\%$ \\
\hline Total deliveries & 662,770 & 610,716 & \\
\hline Maternal age (mean, SD) & 29 & 5.8 & 29 & 5.8 \\
Maternal race and ethnicity $^{\mathrm{a}}$ & & & & \\
\hline Non-Hispanic White & 358,484 & 54.0 & 329,907 & 53.7 \\
\hline Hispanic & 113,322 & 17.1 & 110,212 & 17.9 \\
\hline Non-Hispanic Black & 92,388 & 13.9 & 88,813 & 14.5 \\
\hline Non-Hispanic Asian & 29,777 & 4.5 & 26,706 & 4.3 \\
\hline Non-Hispanic Other & 50,951 & 7.7 & 40,592 & 6.6 \\
\hline Missing & 18,698 & 2.8 & 17,863 & 2.9 \\
\hline Marital status & & & & \\
\hline Married & 320,420 & 48.3 & 289,520 & 47.2 \\
\hline Single & 266,728 & 40.2 & 251,634 & 41.0 \\
\hline Other & 70,424 & 10.6 & 65,876 & 10.7 \\
\hline Unknown & 6,048 & 0.9 & 7,063 & 1.2 \\
\hline Primary payor ${ }^{b}$ & & & & \\
\hline Private & 333,695 & 50.3 & 308,858 & 50.3 \\
\hline Medicaid & 286,231 & 43.1 & 266,696 & 43.4 \\
\hline Self-pay & 14,866 & 2.2 & 11,373 & 1.9 \\
\hline Other & 28,828 & 4.3 & 27,166 & 4.4 \\
\hline Census division ${ }^{\mathrm{b}}$ & & & & \\
\hline East North Central & 104,432 & 15.7 & 99,504 & 16.2 \\
\hline East South Central & 49,242 & 7.4 & 46,184 & 7.5 \\
\hline Middle Atlantic & 85,016 & 12.8 & 75,175 & 12.2 \\
\hline Mountain & 47,630 & 7.2 & 44,612 & 7.3 \\
\hline New England & 12,040 & 1.8 & 12,186 & 2.0 \\
\hline Pacific & 80,993 & 12.2 & 62,148 & 10.1 \\
\hline South Atlantic & 165,558 & 25.0 & 162,799 & 26.5 \\
\hline West North Central & 40,820 & 6.2 & 37,538 & 6.1 \\
\hline West South Central & 77,889 & 11.7 & 73,947 & 12.0 \\
\hline Hospital location ${ }^{\text {d }}$ & & & & \\
\hline Urban & 581,946 & 87.7 & 538,636 & 87.7 \\
\hline Rural & 81,674 & 12.3 & 75,457 & 12.3 \\
\hline Maternal comorbidities & & & & \\
\hline Hypertensive disorders of pregnancy & 99,324 & 15.0 & 101,013 & 16.5 \\
\hline Diabetes (T1/T2, gestational, other) & 63,989 & 9.6 & 68,432 & 11.1 \\
\hline$\quad$ Type 1 or type 2 diabetes & 7,419 & 1.1 & 7,630 & 1.2 \\
\hline Obesity & 55,738 & 8.4 & 59,852 & 9.8 \\
\hline
\end{tabular}

Abbreviations: $\mathrm{SD}=$ Standard deviation; $\mathrm{T} 1 / \mathrm{T} 2=$ Type 1 or Type 2

a. Maternal race and ethnicity collected separately and combined; Women with records indicating Hispanic ethnicity were considered Hispanic, regardless of recorded or missing race. Women missing Hispanic ethnicity or categorized as non-Hispanic were classified as nonHispanic and assigned their recorded race.

b. Private payor includes managed care and commercial indemnity

c. East North Central: Indiana, Illinois, Michigan, Ohio, Wisconsin; East South Central: Alabama, Kentucky, Mississippi, Tennessee; Middle Atlantic: New Jersey, New York, 
Pennsylvania; Mountain: Arizona, Colorado, Idaho, New Mexico, Montana, Utah, Nevada, Wyoming; New England: Connecticut, Maine, Massachusetts, New Hampshire, Rhode Island, Vermont; Pacific: Alaska, California, Hawaii, Oregon, Washington; South Atlantic: Delaware, District of Columbia, Florida, Georgia, Maryland, North Carolina, South Carolina, Virginia, West Virginia; West North Central: Iowa, Kansas, Minnesota, Missouri, Nebraska, North Dakota, South Dakota; West South Central: Arkansas, Louisiana, Oklahoma, Texas

d. The U.S. Census defines an urban area as a territory whose core census block groups or blocks have a population density of at least 1000 people per square mile, and surrounding census blocks have an overall density of at least 500 people per square mile. Rural areas are considered territory outside the definition of urban.

e. Includes chronic hypertension, gestational hypertension, chronic hypertension with superimposed pre-eclampsia, pre-eclampsia, HELLP syndrome, and eclampsia

Table 2. Association between adverse pregnancy outcomes among pregnancies being delivered in April-December 2020 compared to April-December 2019, Premier Healthcare Database Special COVID-19 Release, United States 


\begin{tabular}{|c|c|c|c|c|c|c|}
\hline & 2019 & & 2020 & & & \\
\hline & $\mathrm{n}$ & $\%$ & $\mathrm{n}$ & $\%$ & $\begin{array}{c}\text { Prevalence } \\
\text { ratio } \\
(95 \% \mathrm{CI})\end{array}$ & $\begin{array}{l}\text { Adjusted } \\
\text { prevalence } \\
\text { ratio }(95 \% \\
\text { CI) }\end{array}$ \\
\hline Total & 663,620 & & 614,093 & & & \\
\hline \multicolumn{7}{|l|}{$\begin{array}{l}\text { Adverse maternal } \\
\text { outcomes }\end{array}$} \\
\hline $\begin{array}{l}\text { Intensive care unit } \\
\text { admission }^{\mathrm{a}}\end{array}$ & 10,964 & 1.7 & 9,480 & 1.5 & $\begin{array}{r}0.93 \\
(0.91 \\
0.96)\end{array}$ & $\begin{array}{r}0.90(0.87, \\
0.92)^{\mathrm{a}}\end{array}$ \\
\hline In hospital death & 33 & $\begin{array}{r}5 \text { per } \\
100,000\end{array}$ & 53 & $\begin{array}{r}9 \text { per } \\
100,000\end{array}$ & $\begin{array}{r}1.74 \\
(1.12, \\
2.68)\end{array}$ & $\begin{array}{r}1.68(1.09, \\
2.59)^{\mathrm{b}}\end{array}$ \\
\hline \multicolumn{7}{|l|}{ Pregnancy outcomes } \\
\hline Livebirth & 659,663 & 99.4 & 610,303 & 99.4 & $\begin{array}{r}1.00 \\
(1.00, \\
1.00)\end{array}$ & $\begin{array}{r}1.00(1.00, \\
1.00)^{\mathrm{C}}\end{array}$ \\
\hline Stillbirth & 3,957 & 0.6 & 3,790 & 0.6 & $\begin{array}{r}1.03 \\
(0.98 \\
1.07)\end{array}$ & $\begin{array}{r}1.02(0.98, \\
1.07)^{\mathrm{C}}\end{array}$ \\
\hline Preterm birth & 61,101 & 9.2 & 56,158 & 9.1 & $\begin{array}{r}0.99 \\
(0.98, \\
1.00)\end{array}$ & $\begin{array}{r}0.96(0.95, \\
0.97)^{\mathrm{C}}\end{array}$ \\
\hline
\end{tabular}

Mode of delivery

Vaginal

$458,464 \quad 69.1 \quad 419,869$

68.4

0.99

$69,464.1969$

$(0.98$,

0.99 )

1.03

Cesarean

205,156

$30.9 \quad 194,224$

31.6

(1.03,

1.04)

$1.00(1.00$,

Cesarean delivery

characteristics

..with PROM

15,231

$7.4 \quad 15,083$

7.8

1.05

(1.02,

1.07)

1.05

..with prolonged labor

2,109

$1.0 \quad 2,105$

1.1

(0.99,

..with attempted

forceps or vacuum

1,176

$0.6 \quad 1,149$

0.6

$1.12)$

1.03

..with augmentation or

induction of labor

9,082

$4.4 \quad 8,777$

(0.95,

1.12)

1.02

$1.06(1.04$,

$1.01(1.00$ $1.01)^{\mathrm{C}}$

SMFM cesarean delivery designation

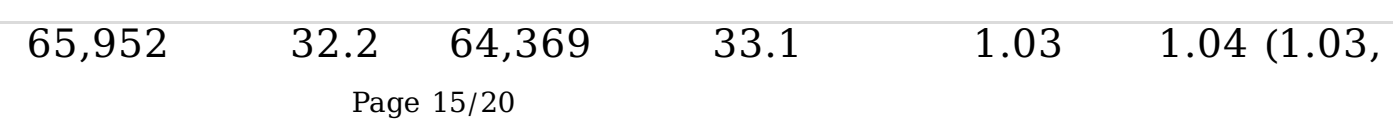


High-risk ${ }^{\mathrm{e}}$

139,204

$67.9 \quad 129,855$

(0.98,

$0.98(0.98$,

....Due to maternal

66.9

0.99 )

1.11

factors ${ }^{\mathrm{e}}$

4,609

2.3

4,838

(1.06,

1.07 (1.03,

1.15)

1.01

....Due to preterm

birth

26,944

$13.1 \quad 25,667$

(0.99,

1.02)

1.03

(0.93,

....Due to stillborn

727

0.4

719

1.15)

1.00

....Due to

26,555

$12.9 \quad 25,204$

(0.99,

malpresentation

26,555

1.02)

1.01

....Due to fetal factors

234

0.1

(0.84,

....Due to

uterine/placental

factors

105,848

$51.6 \quad 97,937$

224

1.21)

0.1

0.97

(0.97,

$0.98)$

1.09

....Due to conduct of

labor

2,553

$1.2 \quad 2,642$

(1.04,

1.15)

$1.12)^{\mathrm{C}}$

$0.98(0.96$, $0.99)^{\mathrm{C}}$ 
Table 3. Association between delivery length of stay among pregnancies being delivered in April-December 2020 compared to April-December 2019, Premier Healthcare Database Special COVID-19 Release, United States

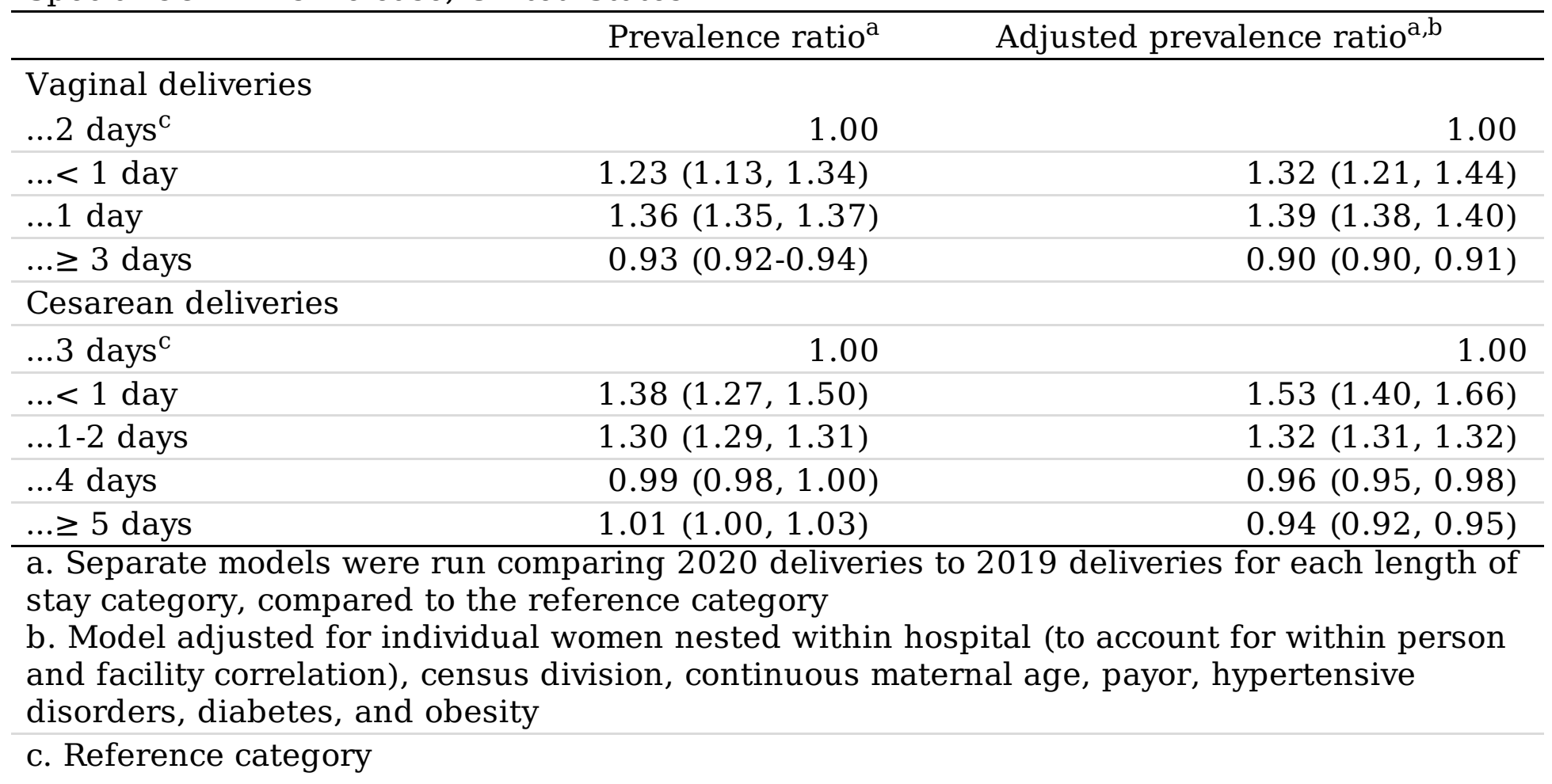

\section{Figures}




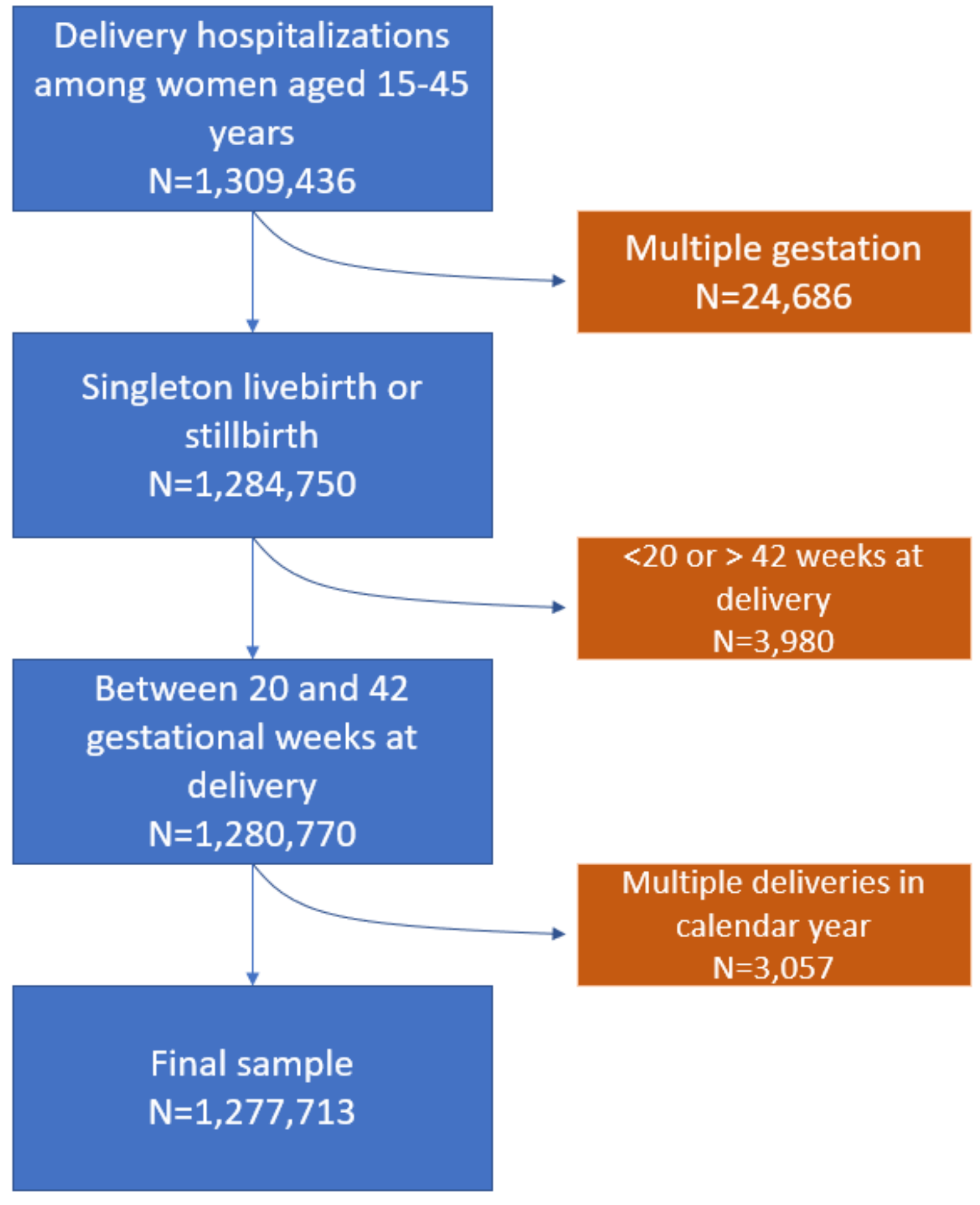

Figure 1

Inclusion and exclusion criteria for the analytic sample of deliveries occurring April-December 2019 and 2020, Premier Healthcare Database Special COVID-19 Release, United States 
Length of delivery hospitalization stay among women with vaginal deliveries, 2019 ( $n=458,464)$ and $2020(n=419,869)$

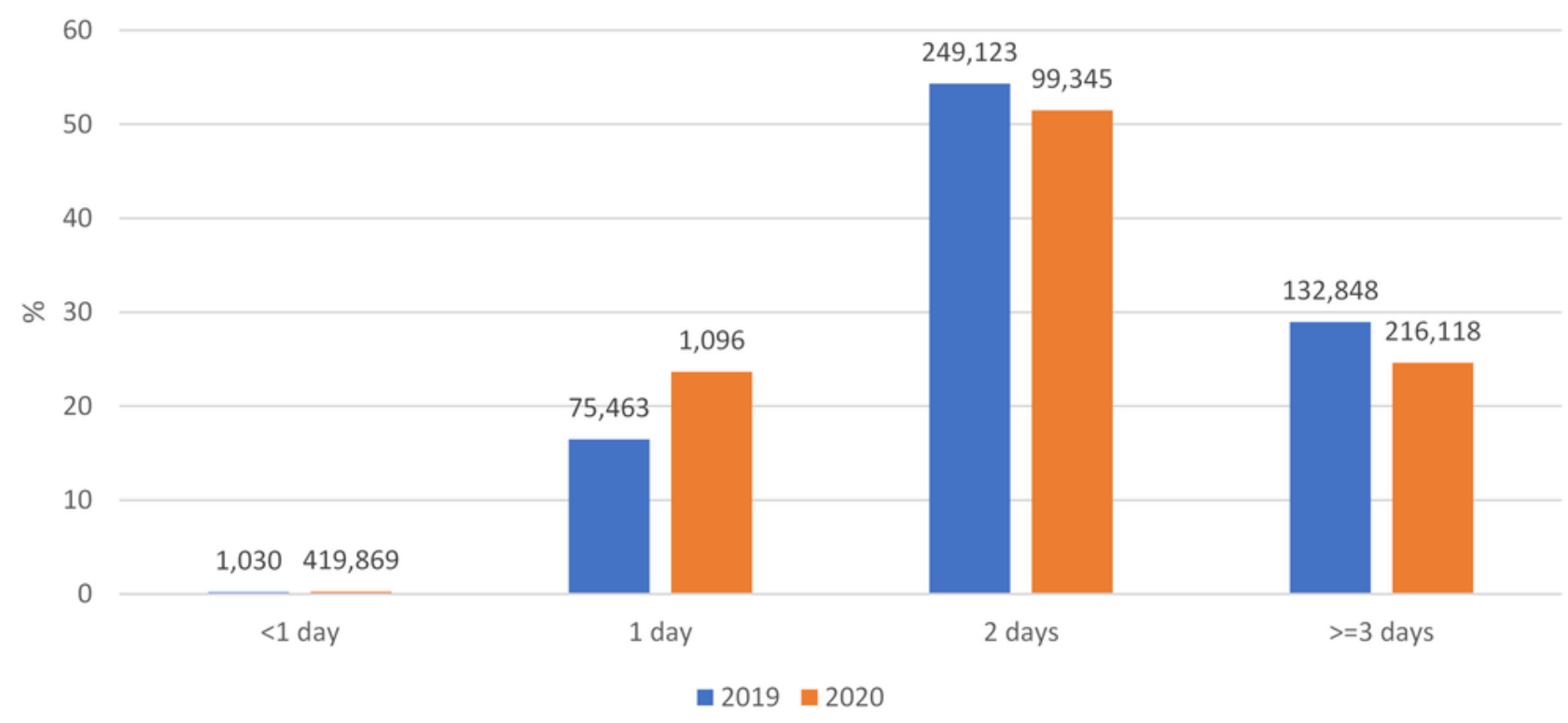

Length of delivery hospitalization stay among women with cesarean deliveries, 2019 $(n=205,159)$ and $2020(n=194,224)$

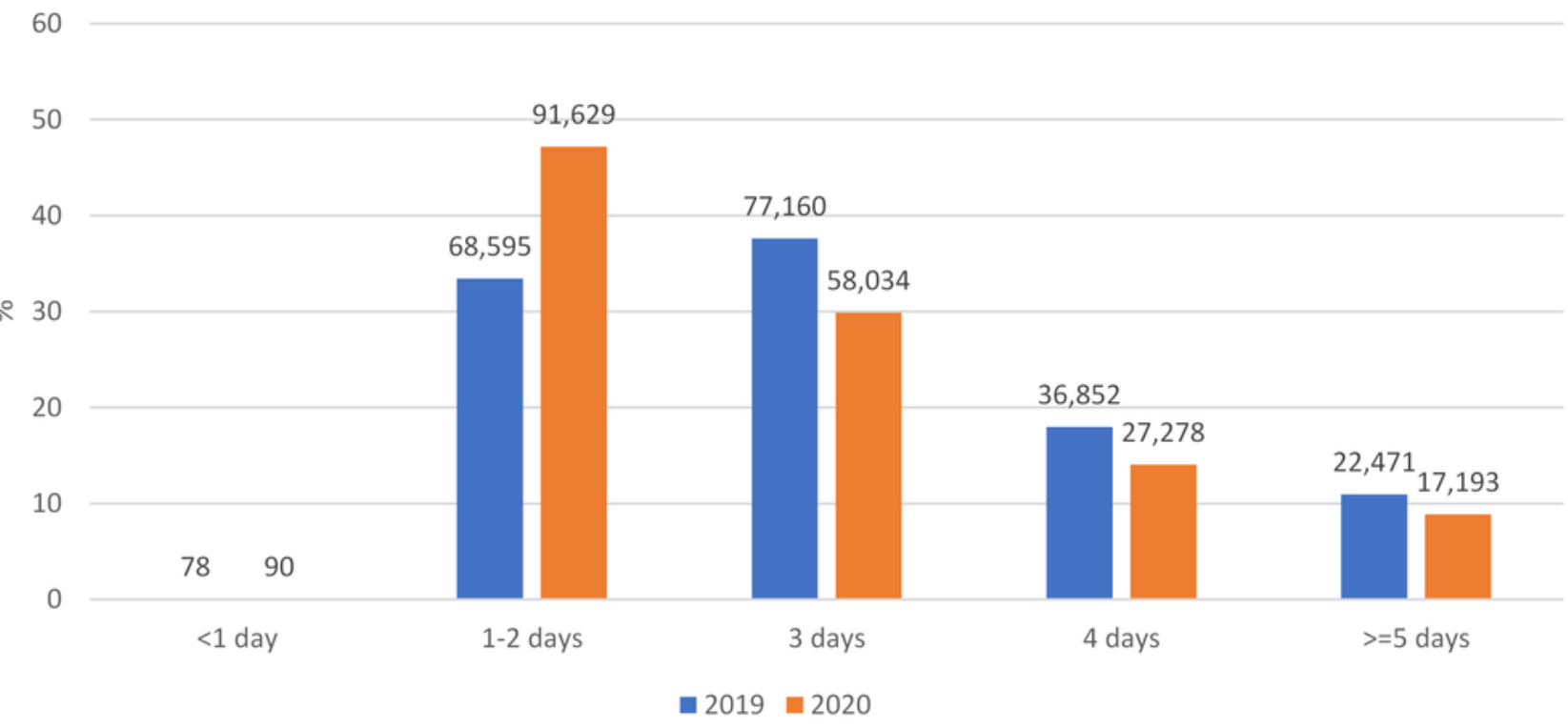

\section{Figure 2}

Length of delivery hospitalization stay among women by mode of delivery, April-December 2019 and 2020, Premier Healthcare Database Special COVID-19 Release, United States

\section{Supplementary Files}


This is a list of supplementary files associated with this preprint. Click to download.

- AppendixPremiermaternalpregoutcomesResSq20210929.docx 